MZ-TH/98-29

July 1998

\title{
Only Three
}

\section{Gabriela Barenboim* and Florian Scheck ${ }^{\dagger}$}

\author{
Institut für Physik - Theoretische Elementarteilchenphysik \\ Johannes Gutenberg-Universitât, D-55099 Mainz, Germany
}

\begin{abstract}
It is shown that it is possible to account for all the experimental indications for neutrino oscillations with just three flavours. In particular we suggest that the atmospheric neutrino anomaly and the LSND result can be explained by the same mass difference and mixing. Possible implications and future test of the resulting mass and mixing pattern are given.
\end{abstract}

*gabriela@thep.physik. uni-mainz.de

${ }_{\dagger}^{\dagger}$ Scheck@dipmza.physik.uni-mainz.de 


\section{Introduction}

Currently there are three pieces of evidence which suggest that neutrinos may have non zero mass differences and nontrivial mixings. These are the anomalies observed in the solar neutrino flux [1], in atmospheric neutrino production [2, 3] and in neutrino beams from accelerators and reactors [4]. The conventional approach in analyzing any single experiment consists in parametrizing the neutrino anomaly in terms of oscillations between two neutrino mass states only. Clearly, this assumption is too restrictive when one sets out to fit several anomalies simultaneously and a consistent mixing scheme involving at least three lepton generations is mandatory [5]. In fact, a two-generation parametrization of a given experimental result may lead to erroneous conclusions about the magnitude of the squared mass differences and/or mixing matrix elements.

In this note we address the question whether all extent observations can be explained simultaneously in terms of three neutrino mass eigenstates which mix and oscillate. In contrast to earlier fits which seemed to suggest that one needed at least four generations [6] we show that one can accommodate all experimental findings in a setting with just three flavours. We find essentially two solutions that describe all data and both of which are characterized by large mixing between the three generations. We also show that the need for a fourth generation becomes obsolete because of a significantly different choice of the relevant mass differences. The mixing pattern predicted by these solutions can be subject to further experimental tests and, hence, may be verified or disproven in ongoing or future experiments.

Regarding the mass spectrum of the three neutrinos we assume that it is characterized by two differences of squared masses

$$
\Delta m^{2} \equiv \Delta m_{21}^{2}=m_{2}^{2}-m_{1}^{2} \quad \text { and } \Delta M^{2} \equiv \Delta m_{32}^{2}=m_{3}^{2}-m_{2}^{2}
$$

whose numerical values are rather different. The first of these is chosen in view of the solar neutrino deficit,

$$
10^{-4} e V^{2} \leq \Delta m^{2} \leq 10^{-3} e V^{2}
$$

The lower limit is suggested by the results of Super KAMIOKANDE where a significant effect would be seen only for values at this level or higher. The upper limit is obtained from the CHOOZ reactor experiment [7] which showed that $\Delta \mathrm{m}^{2} \leq 10^{-3} \mathrm{eV}^{2}$.

The second is selected with both the LSND and the atmospheric neutrino data in mind, viz.

$$
\Delta M^{2} \simeq .3 e V^{2}
$$

This choice reflects the values allowed by the LSND experiment, adding to them the constraint obtained from the Bugey reactor [8], $\Delta M^{2}>.2 \mathrm{eV}^{2}$, and the upper limit obtained in searches for oscillations by CDHSW [9] which gave $\Delta M^{2}<.4 e V^{2}$.

With these assumptions on the mass differences the LSND, the solar and the atmospheric neutrino data can be explained by an appropriate choice of the mixing matrix that defines the weak interaction states. 
The neutrino survival and transition probabilities are given by

$$
P(\alpha \rightarrow \beta)=\delta_{\alpha \beta}-4 \sum_{i>j=1}^{3} U_{\alpha i} U_{\beta i} U_{\alpha j} U_{\beta j} \sin ^{2}\left[\frac{\Delta m_{i j}^{2} L}{4 E}\right]
$$

Here the matrix $U=\left\{U_{\alpha i}\right\}$ describes the weak interaction states $\left(\nu_{\alpha}\right)$ in terms of the mass eigenstates $\left(\nu_{i}\right)$. That is,

$$
\nu_{\alpha}=\sum_{i} U_{\alpha i} \nu_{i} .
$$

For the time being we ignore possible $\mathrm{CP}$ violation but return to this point later. As a consequence $U$ is taken to be real. It is parametrized as follows

$$
U=\left(\begin{array}{ccc}
c_{1} c_{3} & s_{1} c_{3} & s_{3} \\
-s_{1} c_{2}-c_{1} s_{2} s_{3} & c_{1} c_{2}-s_{1} s_{2} s_{3} & s_{2} c_{3} \\
s_{1} s_{2}-c_{1} c_{2} s_{3} & -c_{1} s_{2}-s_{1} c_{2} s_{3} & c_{2} c_{3}
\end{array}\right)
$$

In Eq. (3) $L$ denotes the neutrino flight path, i.e. the distance between the neutrino source and the detector, $E$ is the energy of the neutrino in the laboratory system.

\section{The LSND experiment}

The Liquid Scintillation Neutrino Detector (LSND) experiment at Los Alamos reported to have observed appearance of $\bar{\nu}_{e}$ in an initial beam of $\bar{\nu}_{\mu}$ 's [8]. These results were interpreted as evidence for neutrino oscillations.

The probability to observe oscillations is given by

$$
\begin{aligned}
P_{\mathrm{LSND}}= & -4 U_{23} U_{13} U_{21} U_{11} \sin ^{2}\left[\frac{\left(\Delta m^{2}+\Delta M^{2}\right) L}{4 E}\right] \\
& -4 U_{23} U_{13} U_{22} U_{12} \sin ^{2}\left[\frac{\Delta M^{2} L}{4 E}\right]-4 U_{22} U_{12} U_{21} U_{11} \sin ^{2}\left[\frac{\Delta m^{2} L}{4 E}\right]
\end{aligned}
$$

The LSND set up [10] is characterized by $L=30 \mathrm{~m}$ and $36 \mathrm{MeV}<E<60 \mathrm{MeV}$. With our choice of $\Delta m^{2}$, this means that the third term in Eq.(6) and the contribution of $\Delta m^{2}$ in the first term can be neglected. Combining then the first two terms and making use of the unitarity of $U$ we have

$$
\begin{aligned}
P_{\mathrm{LSND}} & \approx 4 U_{23}^{2} U_{13}^{2} \sin ^{2}\left[\frac{\Delta M^{2} L}{4 E}\right] \\
& =4 s_{2}^{2} c_{3}^{2} s_{3}^{2} \sin ^{2}\left[\frac{\Delta M^{2} L}{4 E}\right]
\end{aligned}
$$

Hence, the requirement that the LSND results $P_{\mathrm{LSND}}=.0031 \pm .0011$ (stat) \pm .0005 (syst) be consistent with our choice of parameters requires

$$
s_{2} c_{3} s_{3}=.104,
$$


where we have taken the average energy $(E=42 \mathrm{MeV})$.

Note that if in Eq. (7) one had inserted a very small squared mass difference it would have been impossible to meet the requirement (8). This may have been the reason that led the authors of [6] to conclude that a fourth generation was necessary to explain the Los Alamos data.

\section{$3 \quad$ Atmospheric neutrinos}

The evidence for an anomaly in atmospheric neutrino experiments was pointed out by the Kamiokande collaboration and by the IMB Collaboration [11]. More recently, the Super Kamiokande collaboration reported further and more precise results on the anomaly in atmospheric neutrino fluxes [3].

Oscillations of muon neutrinos into tau neutrinos as presumably observed by Super Kamiokande are given by

$$
\begin{aligned}
P_{\text {Super K }}= & -4 U_{23} U_{33} U_{21} U_{31} \sin ^{2}\left[\frac{\left(\Delta m^{2}+\Delta M^{2}\right) L}{4 E}\right] \\
& -4 U_{23} U_{33} U_{22} U_{32} \sin ^{2}\left[\frac{\Delta M^{2} L}{4 E}\right]-4 U_{22} U_{32} U_{21} U_{31} \sin ^{2}\left[\frac{\Delta m^{2} L}{4 E}\right]
\end{aligned}
$$

Again, with our choice of squared mass differences, the contributions of $\Delta m^{2}$ can be neglected and the first two terms can be combined into a single one, yielding

$$
P_{\text {Super K }}\left(\nu_{\mu} \rightarrow \nu_{\tau}\right) \approx 4 U_{23}^{2} U_{33}^{2} \sin ^{2}\left[\frac{\Delta M^{2} L}{4 E}\right]
$$

Thus by averaging the sine squared factor to one half (which is consistent with the variation in $E$ and $L$ ), we obtain,

$$
P_{\text {Super K }}=2 s_{2}^{2} c_{2}^{2} c_{3}^{4}
$$

There are good reasons to believe that the overall deficit of muon neutrinos observed in Super Kamiokande atmospheric data for zenith angles whith $\cos \theta_{z}>-.6$ is primarily an effect of muon neutrinos oscillating into tau neutrinos. Indeed, this is supported by the smallness of the LSND effect and the non-observation of electron neutrino disappearance in reactor experiments. However, as we are working in a setting of three neutrino generations, oscillations of muon neutrinos into electron neutrinos are not only possible but also mandatory in our scheme. Super Kamiokande should observe them with a probability given by

$$
\begin{aligned}
P_{\text {Super K }}\left(\nu_{\mu} \rightarrow \nu_{e}\right)= & -4 U_{23} U_{13} U_{21} U_{11} \sin ^{2}\left[\frac{\left(\Delta m^{2}+\Delta M^{2}\right) L}{4 E}\right] \\
& -4 U_{23} U_{13} U_{22} U_{12} \sin ^{2}\left[\frac{\Delta M^{2} L}{4 E}\right]-4 U_{22} U_{12} U_{21} U_{11} \sin ^{2}\left[\frac{\Delta m^{2} L}{4 E}\right](12)
\end{aligned}
$$


Again, the terms in $\Delta m^{2}$ are negligible, the first two terms can be combined in a single one and their sine squared factors can be averaged to one half, resulting in

$$
P_{\text {Super K }}\left(\nu_{\mu} \rightarrow \nu_{e}\right) \simeq 2 U_{23}^{2} U_{13}^{2}-4 U_{22} U_{12} U_{21} U_{11} \sin ^{2}\left[\frac{\Delta m^{2} L}{4 E}\right]
$$

If the mixing angles $\theta_{2}$ and $\theta_{3}$ are small, this implies that, for most of the zenith angle range, the atmospheric neutrino anomaly is indeed due to muon neutrinos oscillating into tau neutrinos. We will see below that our fit does indeed favor small values for these angles. Nevertheless, the second term will also be observable in upward going events provided $\Delta m^{2} L /(4 E)$ is larger than or of order one.

Returning to the observations, using that, for zenith angles whose $\cos \theta_{z}>-.6$ $P_{\text {Super K }}=.3$ [3] we derive our second constraint (see Eq.(11]) on the mixing angles,

$$
s_{2}^{2} c_{2}^{2} c_{3}^{4}=.15
$$

\section{Solar neutrinos}

As is well known, the four experiments set up to detect solar neutrinos found significant deviations from the fluxes expected on the basis of the standard solar model [1, 12]. The observed suppression is often interpreted in terms of matter induced resonant oscillation, the MSW effect, by which, on its way through the sun's interior, the initial electron neutrinos are turned into other neutrino species to which the detectors are blind.

With neutrino mass differences as large as those we are considering in this work, the MSW mechanism is not expected to be relevant. Therefore, the survival probability of a solar electron neutrino is given by

$$
\begin{aligned}
P_{\text {solar }}= & 1-4 U_{13}^{2} U_{11}^{2} \sin ^{2}\left[\frac{\left(\Delta m^{2}+\Delta M^{2}\right) L}{4 E}\right] \\
& -4 U_{13}^{2} U_{12}^{2} \sin ^{2}\left[\frac{\Delta M^{2} L}{4 E}\right]-4 U_{12}^{2} U_{11}^{2} \sin ^{2}\left[\frac{\Delta m^{2} L}{4 E}\right]
\end{aligned}
$$

Again, when the corresponding flight length and energy of the neutrinos detected on Earth allow us to average the sine squared terms to one half, this probability is given by

$$
P_{\text {Solar }}=1-2 c_{3}^{2}\left(s_{3}^{2}+s_{1}^{2} c_{1}^{2} c_{3}^{2}\right)
$$

Although the Chlorine, the Gallium and the Kamioka experiments, probing different parts of the solar neutrino spectrum, seem to find somewhat different suppression factors

we summarize their combined information by taking $P_{\text {solar }}=.5$ [12]. This gives us a third equation fixing the mixing angles,

$$
1-2 c_{3}^{2}\left(s_{3}^{2}+s_{1}^{2} c_{1}^{2} c_{3}^{2}\right)=.5
$$




\section{The mixing pattern and its consequences}

Solving Eqs. (8), (14) and (17), we obtain a neutrino mixing pattern that is compatible at the same time with the LSND, the solar and the atmospheric neutrino anomalies. In fact, we find at first four solutions that we show in Table 1, two of which can be ruled out on phenomenological grounds, (see below).

\begin{tabular}{||c|c|c|cc||}
\hline$\theta_{1}$ & $\theta_{2}$ & $\theta_{3}$ & \multicolumn{3}{|c|}{ CKM } \\
\hline 35.5 & 27.3 & 13.1 & $\left(\begin{array}{ccc}.793 & .566 & .226 \\
-.601 & .662 & .447 \\
.103 & -.490 & .865\end{array}\right)$ \\
\hline 40.4 & 64.2 & 6.5 & $\left(\begin{array}{ccc}.757 & .644 & .113 \\
-.360 & .266 & .894 \\
.546 & -.718 & .432\end{array}\right)$ \\
\hline 49.6 & 64.2 & 6.5 & $\left(\begin{array}{ccc}.644 & .757 & .113 \\
-.398 & .204 & .894 \\
.654 & -.621 & .433\end{array}\right)$ \\
\hline 54.5 & 27.3 & 13.1 & $\left(\begin{array}{ccc}.566 & .792 & .226 \\
-.783 & .432 & .447 \\
.257 & -.430 & .865\end{array}\right)$ \\
\hline
\end{tabular}

Table 1: Mixing patterns

At this point we stress that we restricted ourselves to the central values of the experimental data. Clearly, experimental error bars in the inputs will reflect themselves in uncertainties of the resulting parameters. Here, however, our aim was primarily to show that the central values can account essentially for all observations.

It is also important to notice that, like other, previous attempts to explain neutrino phenomena with oscillations, ours is subject to the resolution of possible inconsistencies between experiments. For example, we have assumed that the LSND results is an oscillation effect rather than a background one. However, one should keep in mind that while the LSND collaboration has announced additional evidence [13] KARMEN has not confirmed their result [14] 巴.

The next question is how to distinguish between the four solutions we give in Table 1. The answer is clear: try with reactor experiments. Eq. (15) which we have used for solar neutrinos is also valid for reactor experiments. Although these experiments are rather insensitive to the smaller of the assumed differencies $\left.\Delta \mathrm{m}^{2}\right|_{\max }=10^{-3} \mathrm{eV}^{2}$, they should observe oscillations with $\Delta M^{2}=.3 \mathrm{eV}^{2}$. For values of $E$ and $L$ that allow an averaging of the sine-squared term, we have

$$
P_{\text {reactor }}=1-2 s_{3}^{2} c_{3}^{2}
$$

We therefore predict $P_{\text {reactor }}=.90$ in cases I and IV and $P_{\text {reactor }}=.97$ (cases II and III). This must be compared with the Bugey result of .99 \pm .01 (stat) \pm .005 (sys) and the

\footnotetext{
${ }^{1}$ For our assumed parameters KARMEN should have yielded two or three real events on top of other three background ones
} 
CHOOZ result of $.98 \pm .04$ (stat) \pm .004 (sys). With regard to the experimental errors we can state that all four solutions are in reasonable agreement with the reactor data.

Up to this point all experimental phenomena which are believed to be due to neutrino oscillations, can be understood by the mixing matrices of cases I and IV and by squared mass differences in the range $10^{-4} \mathrm{eV}^{2}<\Delta \mathrm{m}^{2}<10^{-3} \mathrm{eV}^{2}$ and $\Delta M^{2}=.3 \mathrm{eV}$. For reasons to be discussed below, the second and third solution are ruled out on phenomenological grounds. Ours sets of parameters seem to be in reasonable agreement with all the features of neutrino observations, including the recently announced zenith angle modulation of the Super Kamiokande atmospheric neutrino data for upward going events $\left(\cos \theta_{z}<-.6\right)$.

Now, provided the atmospheric muon neutrinos are produced at almost twice the rate of the electron neutrinos, the double ratio for these upward going events can be taken to be

$$
\begin{aligned}
R & =\frac{\left(\frac{N_{\mu}}{N_{e}}\right)_{\text {observed }}}{\left(\frac{N_{\mu}}{N_{e}}\right)_{\text {no oscillation }}} \\
& =\frac{1}{2}\left(\frac{2 P_{\mu \mu}+P_{e \mu}}{P_{e e}+2 P_{\mu e}}\right)
\end{aligned}
$$

Assuming $L$ to be sufficiently large so that all oscillation factors average to one half we obtain $R_{\text {large }} \mathrm{L}=.44$ (case I) and .53 (case IV) to be compared with the experimental value $R_{\exp }=.41 \pm .04$ at the largest zenith angles. For cases II and III this double ratio reaches values as large as one and it is for that reason that we should discard them.

As a more detailed test of our assumptions we have also calculated the ratio (19) as a function of $L / E$, i.e. without averaging the oscillation factors. Figs. 1 and 2 show the comparison with the zenith angle dependence as reported by the Super-Kamiokande group [3], for the two limiting values of $\Delta m^{2}$, Eq. 1. Fig. 1 corresponds to solution I, Fig. 2 to solution IV of Table 1. The figures show that the observed variation of $R$ with zenith angle, or oscillation length, is well described in either case; the comparison does not allow, as yet, to discriminate between cases I and IV.

On top of that, the three neutrino mixing scheme predicts sizeable oscillations of electron to tau neutrinos that should be observed by the long baseline neutrino experiments with proposed high intensity muon sources [15]. The probability of such an oscillation in our model, is given by,

$$
P=4 s_{3}^{2} s_{2}^{2} c_{3}^{2} \sin ^{2}\left[\frac{\Delta M^{2} L}{4 E}\right]
$$

which is well above the expected sensitivity. The measurement of electron to tau oscillations in such long baseline experiments would test the three neutrino model.

When the mixing matrix is allowed to have a $\mathrm{CP}$ violating phase, the $\mathrm{CP}$ violating off diagonal probability differences are given by [16]

$$
\begin{aligned}
\Delta P & =P(\bar{\mu} \rightarrow \bar{e})-P(\mu \rightarrow e)=-(P(\bar{\mu} \rightarrow \bar{\tau})-P(\mu \rightarrow \tau)) \\
& =P(\bar{e} \rightarrow \bar{\tau})-P(e \rightarrow \tau) \\
& =-4 J \sin \delta\left[\sin \Delta_{21}+\sin \Delta_{32}+\sin \Delta_{31}\right]
\end{aligned}
$$


where $J$ is the Jarlskog invariant [17], $J=s_{1}^{2} c_{1} s_{2} c_{2} s_{3} c_{3}$ and

$$
\Delta_{i j}=\Delta m_{i j}^{2} L / 2 E
$$

with $\delta$ being the $\mathrm{CP}$ violating phase in the mixing matrix. In this formula, the effects of the mixing are conveniently separated from the effects of the masses, appearing in the $\Delta_{i j}$.

Within our model, $J \leq .07$ and $\left[\sin \Delta_{21}+\sin \Delta_{32}+\sin \Delta_{31}\right] \simeq \mathcal{O}(1)$ for $L / E=$ $730 \mathrm{~km} / 10 \mathrm{GeV}$ (relevant for MINOS) and also for $L / E=730 \mathrm{~km} / 25 \mathrm{GeV}$, (relevant for ICARUS). Hence, $\Delta P$ can be as large as .07 (for these parameters, matter effects are negligible 18]).

We have more implications. The neutrino mass spectrum implied by our scenarios is given by,

$$
\begin{aligned}
& m_{1} \simeq m \\
& m_{2} \simeq \sqrt{m^{2}+\Delta m^{2}} \\
& m_{3} \simeq \sqrt{m_{2}^{2}+\Delta M^{2}}
\end{aligned}
$$

There are two limiting cases of interest assuming that the largest mass is in the $\mathrm{eV}$ range.

(i) One is the hierarchical limit, in which $m$ is negligible. Then $m_{2} \simeq 10^{-2} \mathrm{eV} \gg m_{1}$ and $m_{3} \simeq .6 \mathrm{eV}$.

(ii) The second one is the nearly degenerate limit in which

$$
\begin{aligned}
& m_{1} \simeq 1 \mathrm{eV} \\
& m_{2} \simeq 1 \mathrm{eV} \\
& m_{3} \simeq 1.2 \mathrm{eV}
\end{aligned}
$$

In this case the sum of the neutrino masses is $\sum_{i} m_{i} \simeq 3.2 \mathrm{eV}$. The cosmological density parameter associated with neutrinos becomes

$$
\Omega_{\nu}=.011 h^{-2} \sum_{i} m_{i}=.035 h^{-2} \simeq .2
$$

for $h$ of about .5, and the amount of the neutrino dark matter component along with cold dark matter makes for a viable and testable scenario for mixed dark matter [19.

If these neutrinos are Majorana particles, the effective mass $<m_{\nu_{e}}>$ relevant in neutrinoless double beta decay analysis is

$$
<m_{\nu_{e}}>=\sum_{i} U_{e i}^{2} m_{i}
$$

We find that in the case of hierarchical spectrum $<m_{\nu_{e}}>\simeq .14 \mathrm{eV}$ whereas in the degenerate case $\left\langle m_{\nu_{e}}>\simeq 1.5 \mathrm{eV}\right.$ (this could be somewhat smaller when CP phases are taken into account). It is interesting that these values are in the range of what the double beta decay experiments can probe now and in the near future [20]. 


\section{Conclusions}

This analysis shows that, when taking the experimental results at face value, the evidence for neutrino oscillations is substantial. Although none of the existing positive signals is beyond some criticism, their ensemble is quite compelling.

All the available experimental information from accelerator, atmospheric and solar neutrinos is accounted for in the framework of an analysis assuming mixing of just three flavours. The solutions that we found are not significantly contradicted by any existing result, all conflicting evidence being below the two sigma level.

In order to distinguish our analysis from the one with a single oscillation process with a small $\Delta m^{2}$ requires precise measurements of the multi GeV, overhead $\left(\cos \theta_{z} \sim 1\right)$ events at Super Kamiokande. Our pattern of mixings predicts, in $R \sim .69$ (85) for case I (case IV) while the single oscillation one requires $R \sim 1$.

Perhaps most important is our prediction that muon neutrino to electron neutrino oscillations should be confirmed by an observation of an excess of high energy electrons such us upgoing shower events. An analysis of the proposed, and perhaps already observed,

mixing pattern in terms of mass matrices of charged and neutral leptons along the lines of ref. [21] is in progress and will be published elsewhere.

\section{Acknowledgements}

We are very grateful to J.Bernabeu and S. Petcov for enlightening discussions. A post-doctoral fellowship of the Graduiertenkolleg "Elementarteilchenphysik bei mittleren und hohen Energien" of the University of Mainz is also acknowledged.

\section{References}

[1] B.T.Cleveland et al. , Nucl. Phys. B38 (Proc. Suppl.) (1995) 47;

Y.Fukuda et al. , Phys. Rev. Lett. 77 (1996) 1683;

W.Hampel et al. , Phys. Lett. B388 (1996) 384;

J.N.Abdurashitov et al. , Phys. Rev. Lett. 77 (1996) 4708;

J.N.Bahcall and M.H.Pinsonneault, Rev. Mod. Phys. 67 (1995) 781;

J.N.Bahcall, S.Basu and M.H.Pinsonneault, astro-ph/9805135.

[2] K.S.Hirata et al. , Phys. Lett. B280 (1992) 146;

R.Becker-Szendy et al. , Phys. Rev. D46 (1992) 3270.

[3] Y.Fukuda et al. , hep-ex/9803006, 9805006, 9805021, and 9807003.

[4] C.Athanassopoulos et al. , Phys. Rev. Lett. 75 (1995) 2650; ibid. 77 (1996) 3082.

[5] P.Harrison, D.Perkins and W.Scott, Phys. Rev. B349 (1995) 137;

G.Conforto et al. , Astroparticle Phys. 5 (1996) 147;

A.Acker and S.Pakvasa, Phys. Lett. B397 (1997) 209; 
G.L.Fogli et al. , Phys. Rev. D56 (1997) 4365;

R.P.Thun and S.Mckee, hep-ph/9806534;

V.Barger, T.J.Weiler and K.Whisnant, hep-ph/9807319.

[6] S.M.Bilenky, C.Giunti and W.Grimus, Nucl. Phys. B666 (Proc. Suppl.) (1998) 404; ibid. hep-ph/9711311.

[7] M.Apollonio et al. , Phys. Lett. B420 (1998) 397.

[8] B.Achkar et al. , Nucl. Phys. B434 (1995) 503.

[9] F.Dydak et al. , Phys. Lett. B134 (1984) 281.

[10] C.Athanassopoulos et al. , Phys. Rev. C54 (1996) 2685.

[11] K.S.Hirata et al. , Phys. Lett. B280 (1992) 146;

D.Casper et al., Phys. Rev. Lett. 66 (1991) 2561.

[12] See for example, G.Conforto et al., talk at Neutrino 98, Tkayama, Japan, June 1998.

[13] C.Athanassopoulos et al. , nucl-ex/9709006.

[14] B.Zeitnitz, talk at Neutrino 98, Tkayama, Japan, June 1998.

[15] S.Geer, hep-ph/9712290;

V.Barger, K.Whisnant and T.J.Weiler, Phys. Lett. B427 (1998) 97.

[16] V.Barger, K.Whisnant and R.J.N.Phillips, Phys. Rev. Lett. 26 (1980) 2084.

[17] C.Jarlskog, Phys. Rev. Lett. 55 (1985) 1039.

[18] M.Tanimoto, Phys. Rev. D55 (1997) 322;

J.Arafune and J.Sato, Phys. Rev. D55 (1997) 1653.

[19] J.Primak et al. , Phys. Rev. Lett. 74 (1995) 2160;

L.Kofman et al. , Ap. J. 470 (1996) 102;

S.Doddelson, E.Gates and M.Turner, Science 274 (1996) 69.

[20] M.K.Moe, Int. Jour. Mod. Phys. E2 (1993) 507;

R.S.Raghavan, Phys. Rev. Lett. 72 (1994) 1411.

[21] R. Ha̋ußling, M. Paschke and F. Scheck, Phys. Lett. B417 (1998) 312 
Case I

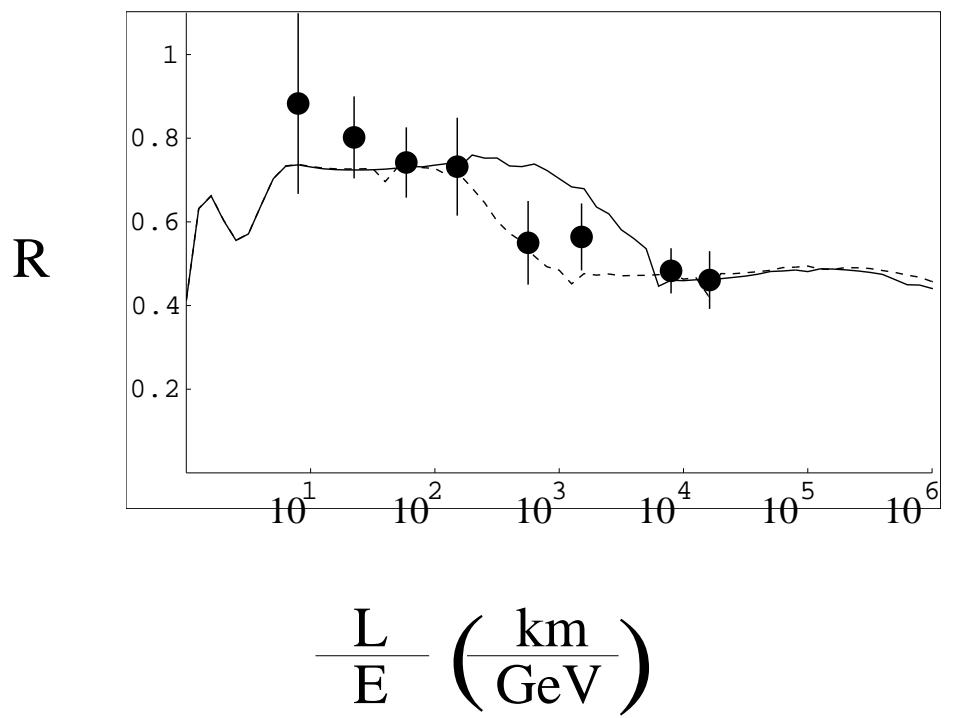

Figure 1: The ratio $R$, Eq. (19), as a function of oscillation length over neutrino energy, $L / E$, in $\mathrm{km} / \mathrm{GeV}$, calculated for case I and compared to Super Kamiokande data. The solid line corresponds to the lower, the dashed line to the upper limit on $\Delta m^{2}$ of Eq. 1.

\section{Case IV}

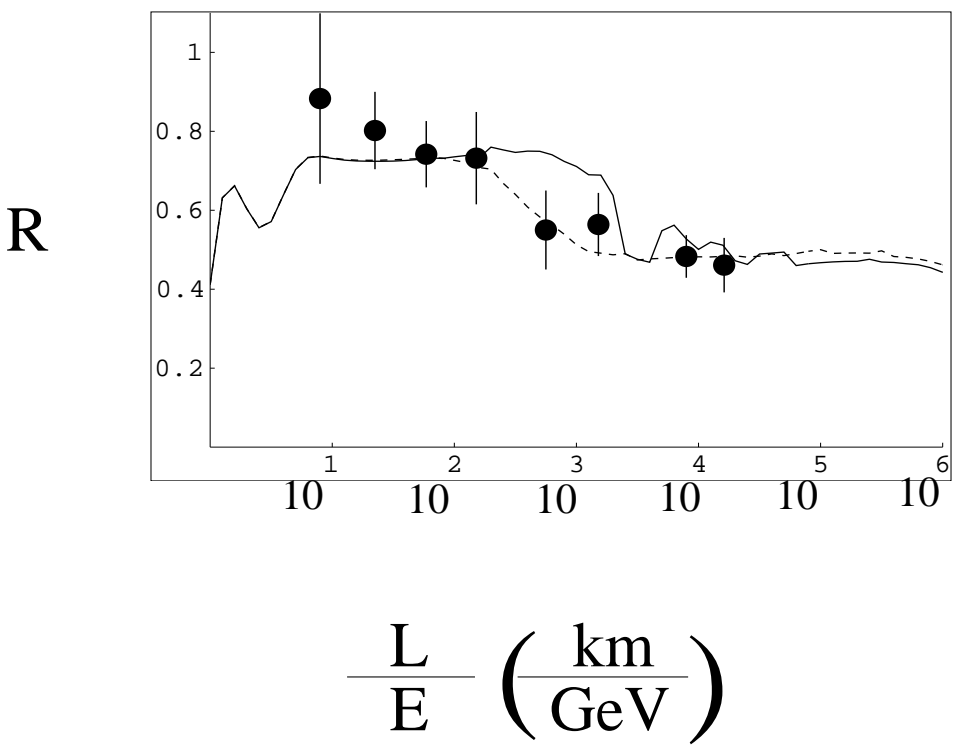

Figure 2: Same as Fig. 1 for case IV. 